\title{
Feature Extraction and Real-Time Recognition of Hand Motion Intentions from EMGs via Artificial Neural Networks
}

\author{
Artemiy Oleinikov, Berdakh Abibullaev, Member, IEEE, Almas Shintemirov, Member, IEEE, \\ Michele Folgheraiter*, Member, IEEE,
}

\begin{abstract}
Electromyography (EMG) signal analysis is one of the key determinants of the effectiveness of prosthetic devices. Modern researchers provide various methods of detection of different hand movements and postures. In this work, we examined the possibility to produce efficient detection of hand movement to a specific posture with the minimum possible number of electrodes. The data acquisition is produced with 1 channel BiTalino EMG sensor based on bipolar differential measurement. Using feature extraction and artificial neural network we achieved $82 \%$ of offline classification accuracy for 8 hand motions and $91 \%$ accuracy for 6 hand motions based on 200ms of EMG signal. Also, the motion detection algorithm was developed and successfully tested that allowed to implement the algorithm for real-time classification that showed sufficient accuracy for 2 and 4 motion classes cases.
\end{abstract}

Index Terms-EMG, Hand, posture detection, ANN, BiTalino, Blender.

\section{INTRODUCTION}

$\mathbf{T}$ HE human hand represents a fundamental part of the body that is involved in nearly every physical activity. The issue of reintegrating people with upper limb amputations or disabilities in our society is of fundamental importance. The latest researches in the field of active arm prosthesis propose several methods of control for the device. Mainly, the solutions are divided into two groups invasive and non-invasive. For invasive type, there are such techniques as implantable electrodes and muscle reinnervation [1]. Due to the complexity, high restrictions in everyday use and relatively high cost of invasive methods, non-invasive methods presented by surface electrodes are widely used as in academia as in commercial use [1]-[3]. Conversely, usage of surface electrodes varies by their number and placement. To evaluate the efficiency of proposed prosthetic devices it is crucial to account that the human hand has 27 degrees of freedom (DOF) [4]. Apart of detection and classification of motion intention, there is some success was made in term proportional force control [15]. Despite the relevant progress in detecting the desired gestures with sufficient accuracy, the number of hand gestures detectable nowadays is still limited; furthermore, the control of state-of-the-art prosthesis is a choice between proportional

All authors are with the Robotics and Mechatronics department, School of Science and Technology at Nazarbayev University, Astana, Kazakhstan. *-corresponding author: michele.folgheraiter@nu.edu.kz control of few motions and pattern-recognition-based control of many.

The first generations of the active arm prosthesis were based on direct proportional control by the electric activity of residual muscles [1]. One of the first researchers for prosthesis control based on the EMG signal features detection was published in the seventies and it had met computational limitations [5]. With the development of technologies, the computational limitations were overcome, subsequently, various new features were proposed [1]-[3], [6]. At the same time, a new type of detection of myoelectric activity was implemented. Utilizing the High-density electrodes grid it becomes possible to implement Non-Negative Matrix Factorization(NMF) that combines spatial features of signal and regression analysis to construct transformation from EMG signal vector to vector of myoelectric activity [6], [7]. The NMF allows implementing proportional control of several DOFs. The drawback of this method is a high number of electrodes required for the system that is reflected in cost and reliability for a long-term use.

For EMG signal features based control, the main issue is the construction of a classifier. Various methods of features classification are already introduced in the literature. Some of them are - Artificial Neural Network (ANN) [8]-[10], Linear Discriminant Analysis (LDA) [11], fuzzy classifier, Support Vector Machine (SVM) [13], [14], classifiers based on Principal Component Analysis (PCA) [12] and Self-Organizing Map (SOM). For pattern recognition of EMG signals, Neural Network classification has been emphasized for a reason that both linear and non-linear relationships can be represented by the modeled data. Different ANN based classifiers have already been successfully implemented for the task of hand motion and gesture recognition [8]-[10]. ANN classification is also applicable for the work with real-time data recognition, which is the end-goal of the research [3]. Despite such drawbacks of the ANN as relatively large minimal sample size, the possibility of overfitting and black box structure in terms of understanding of underlying processes, it is still an efficient data classification tool. In this research, the ANNbased classifier will be utilized with the intention of improving the result of the work described in [8].

There are numerous types of ANN algorithms, which have their own advantages and drawbacks. A common drawback to all types of ANN is that the complexity of network structure 
Pre-Print Version. To cite correctly this paper please refer to: https://ieeexplore.ieee.org/document/8311527

increases the number of input dimensions [3]. For the research, the neural network is trained using the supervised approach. The trained network used to conduct classification in the real time. In addition, accounting the variation of the motor unit action potential (MUAP) due to subjects individual characteristics of skin conductivity, muscle location, and dimensions, etc., a unique ANN is constructed for each subject [8].

\section{Methods}

\section{A. Experimental setup}

Three electrodes of EMG sensor are attached to the surface of test subjects forearm. It was decided to utilize the location of the electrodes that were described in [8], [11], positive electrode is on top (between extensor digitorum communis and extensor carpi ulnaris) and negative on the bottom (flexor carpi radialis) of the upper part of the forearm, and the ground is on radial bone at elbow. The position of electrodes is shown in "Fig. 1". EMG Sensor provides bipolar differential measurement in the range of $\pm 1.65 \mathrm{mV}$ and amplifies it with the Gain of 1000. The resolution of the analog to digital converter is $10 \mathrm{bit}$ and the sample rate of the board is $1 \mathrm{kHz}$. Acquired data are stored in the local buffer until a request from PC through the Bluetooth interface. On the PC side, it was implemented the synchronous acquisition of samples with period $100 \pm 2 \mathrm{~ms}, 100$ data points per sample.

Setup showed limitations of observability and distinguishability of finger movements, so only hand movements will be evaluated.

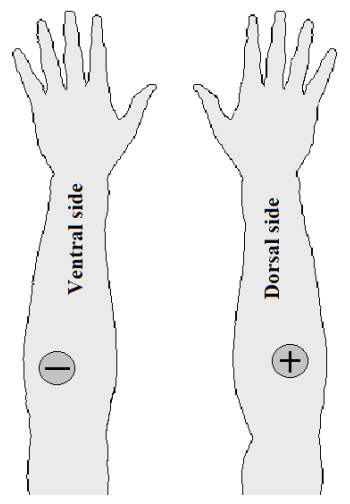

Fig. 1. Electrodes location. Positive electrode locates on the upper part of the forearm on the dorsal side. The negative - on the ventral side. Electrodes have muscle specific location.

\section{B. Detection of the beginning of the motion}

In order to proceed with motion classification, it is necessary to extract EMG signal of the first 200ms of the motion [8], [11]. Because we are acquiring data each $100 \mathrm{~ms}$ it was decided to implement algorithm which detects signal oscillations of high amplitudes within 1 sample of 100 data points. The following formula is implemented:

$$
\begin{array}{r}
k(\text { end }+1)=\operatorname{sum}\left(\operatorname { a b s } \left(\operatorname { d i f } f \left(E M G_{-} \text {data }(\ldots\right.\right.\right. \\
\text { length } \left.\left.\left(E M G_{-} \text {data }\right)-99: \operatorname{length}\left(E M G_{-} \text {data }\right)\right)\right) \ldots \\
>0.07)>5 \& \& \operatorname{sum}(k(\text { end }-4: \text { end }))==0 ;
\end{array}
$$

Where $\mathrm{k}$ - binary vector that stores beginning of the motion in sample domain, EMG_data - vector of raw EMG signal in $\mathrm{mV}$. The time delay of $500 \mathrm{~ms}$ introduced to wait when the motion ends.

\section{Motion classification}

Recent researches showed relatively high accuracy of motion classification based on extracted features [8]. To simplify the resultant network, it was decided not to use raw EMG signal as input to ANN, as in [10]. On the first phase of work the following time-based features were extracted from the $100 \mathrm{~ms}$ samples:

1) Mean Absolute Value (MAV):

$$
M A V_{i}=\frac{1}{N} \sum_{k=1}^{N}\left|x_{k}\right|
$$

2) Difference of Mean Absolute Value (DMAV):

$$
D M A V_{i}=M A V_{i}-M A V_{i-1}
$$

3) Zero Count (ZC): Number of times wave crosses zero.

$$
Z C_{i}=\left\{\begin{array}{l}
\text { If } \operatorname{sign}\left(x_{k+1}\right) \neq \operatorname{sign}\left(x_{k+1}\right) \\
A N D\left|x_{k}-x_{k+1}\right| \geq 0.004(m V) \\
\text { increment by } 1
\end{array}\right.
$$

4) Slope Sign Change (SSC): The SSC is incremented by 1 in two cases:

$$
\begin{aligned}
& \text { 1) } x_{k}>x_{k-1} A N D x_{k}>x_{k+1} A N D \ldots \\
& \left|x_{k}-x_{k+1}\right| \geq 0.004(m V) \\
& 2) x_{k}<x_{k-1} A N D x_{k}<x_{k+1} A N D \ldots \\
& \left|x_{k}-x_{k-1}\right| \geq 0.004(m V)
\end{aligned}
$$

5) Waveform Length (WL):

$$
W L_{i}=\sum_{k=1}^{N}\left|x_{k}-x_{k-1}\right|
$$

6) Root Mean Square (RMS):

$$
R M S_{i}=\sqrt{\frac{1}{N} \sum_{k=1}^{N} x_{k}^{2}}
$$

7) Variance (VAR):

$$
V A R_{i}=\frac{1}{N-1} \sum_{k=1}^{N} x_{k}^{2}
$$

These features require relatively low computational time and allow us to decrease the number of inputs for future ANN. At the same time, there is a possibility to utilize frequency domain using Fast Fourier Transform. There are three features will be used: mean frequency, frequency of maximum amplitude and value of amplitude of that frequency. 
Fast Fourier Transform (FFT):

$$
X_{k}=\sum_{n=0}^{N-1} x_{n} e^{-i 2 \pi k n / N} \quad k=0, \ldots, N-1
$$

In some studies [3], authors argue that Daubechies wavelet function (db2, db4, db6, db44 and db45) at decomposition level 4 is the most suitable for Motor Unit Action Potential detection. The general formula of Continuous Wavelet Transform shown below:

$$
\psi(a, b)=\frac{1}{\sqrt{|a|}} \psi\left(\frac{t-b}{a}\right)
$$

$\psi(\mathrm{t})$ is the band-pass function, $\mathrm{a}$ - scale, $\mathrm{b}$ - time location. In addition the autocorrelation function were utilized:

$$
R_{y} y(l)=\sum_{k=1}^{N} y_{n} * y_{n-l}
$$

$\mathrm{R}$ - autocorrelation coefficient, y - real-valued EMG data.

\section{Analysis}

1) Case 1: 100ms, time-domain features only.: The analysis of the system was started with the implementation of 7 timedomain features. It was acquired 507 motion related samples of features for such motions as hand closing/opening, hand flexion/extension, pronation/supination and flexion/extension (at least 50 samples per motion). The samples were selected and labeled based on the Waveform length feature peaks. The WL based labeling was chosen empirically, however, additional manual adjustments of the data set applied to avoid mislabeling. The approach for the labeling was to identify the beginning of the Waveform length peak that corresponds to high amplitude oscillations of the EMG signal. Sample output of the labeling script shown in "Fig. 2". In the design of classification system, we used Pattern recognition ANN. The scaled conjugate gradient with back propagation training method is used together with means squared error function for error analysis. The input for the network was one sample of 7 time-domain features and the output is 8 motion classes. The network consists of one hidden layer and one output layer. [8] states that one layer of hidden neurons works as an ideal approximator. For the output layer neurons, SoftMax function is used. For the hidden layer, it was checked 3 different neurons functions: linear, sigmoid and tangential sigmoid. The different numbers of hidden neurons were tested in the range from 15 to 50 with the step size of 5 . Both layers of network are with biases. Samples were divided randomly to form 3 datasets for training, validation, and testing in proportion $70 \% / 15 \% / 15 \%$. In this case, we were classifying motion based on $100 \mathrm{~ms}$, the best results were observed for the hidden layer of 30 neurons with tangential sigmoid function. Best observed accuracy was $40 \%$.

2) Case 2: 200ms, time-domain and Fourier transform features.: In order to improve classification results, it was decided to use features of two consecutive samples, which together correspond to first $200 \mathrm{~ms}$ of motion. Moreover, it
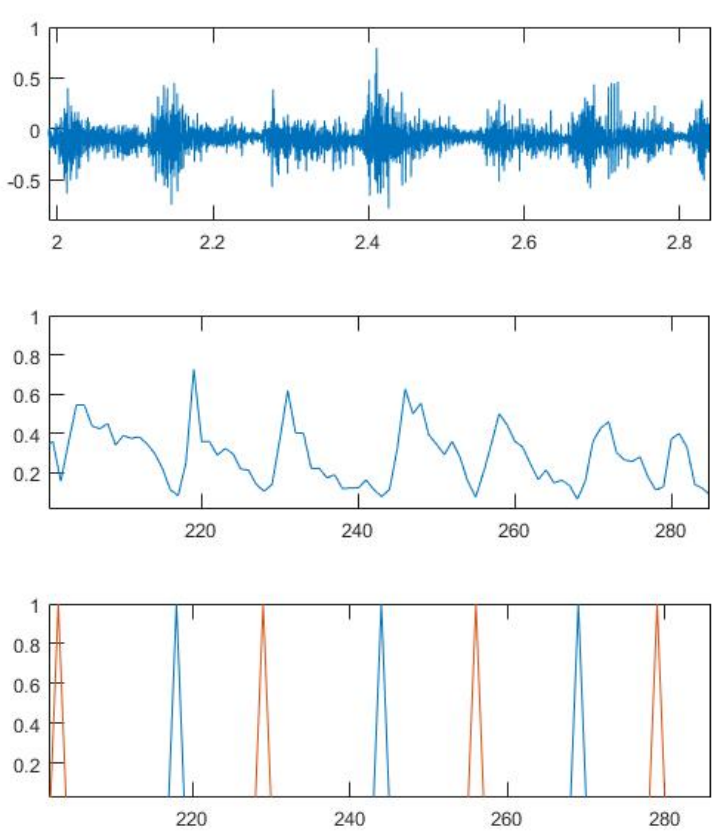

Fig. 2. From top to bottom: Raw signal (mV, x-axis - ms), Waveform length Feature vector (x-axis - samples), two binary vectors present the beginning of motions (x-axis - samples).

was chosen to implement frequency domain features. For the second iteration, we have implemented 7-time domain features described above and 3 FFT based features. It is the frequency with maximum amplitude, the amplitude value of that frequency, amplitude value of mean frequency.

There were conducted 3 data acquisition sessions with 560 samples per session, 70 samples for each of 8 classes. To eliminate the features with the lowest impact to classification the naive strategy was applied. By visual comparison of mean value and standard deviation of features for specific motions, we eliminated SSC, RMS, and Variance. The underlying concept is that specific feature should diverge higher than standard deviation at least for one class. Resultant numbers of inputs for ANN was reduced to 14 (4 Time domain and 3 Frequency domain for 2 samples). For further improvement, we also looked at the autocorrelation, $\mathrm{db} 4$ and $\mathrm{db} 45$ wavelet transforms. By using the same approach, it was defined that standard wavelet transform functions do not have observable distinguishability. For autocorrelation, it is only 2 values out of 21 produced by MATLAB build in function that diverges enough to be accounted. However, by implementing autocorrelation we got just 1-2\% accuracy increase, while its computational complexity in this specific implementation is 10 times higher than for all other features together. Because the computation time for the features with autocorrelation $(110 \mathrm{~ms})$ is higher than time delay between samples, it was decided to eliminate it.

For the ANN we had 14 inputs, hyperbolic tangent sigmoid transfer function for hidden neurons, and SoftMax for output neurons function. Input and hidden layers are with biases. The 
efficiency of the neural network was tested for the different numbers of hidden neurons in the range from 15 to 50 with the step size of 5 . The best accuracy was observed at 25 neurons. In addition, 3 different error measurement functions were tested: sum squared error (SSE), mean squared error (MSE), mean squared error with regularization (MSEREG). MSEREG measures network performance as the weighted sum of two factors: the mean squared error and the mean squared of weight and bias values. Results of this test can be seen in Table I. By analyzing all the combinations of samples, it is reasonable to state that none of the functions showed the significant difference in performance, and based on the training time it was decided to select SSE error functions as the fastest one.

TABLE I

THE ACCURACY OF CLASSIFICATION FOR DIFFERENT ERROR FUNCTION AND DIFFERENT COMBINATIONS OF DATA SAMPLES.

\begin{tabular}{|c|c|c|c|}
\hline & \multicolumn{3}{|c|}{ Error function } \\
\cline { 2 - 4 } & MSEREG & $\boldsymbol{M S E}$ & $\boldsymbol{S S E}$ \\
\hline Day1 & $60.5 \%$ & $60.9 \%$ & $59.5 \%$ \\
\hline Day2 & $56.1 \%$ & $53.4 \%$ & $53.0 \%$ \\
\hline Day3 & $57.1 \%$ & $56.4 \%$ & $57.7 \%$ \\
\hline Day1+2 & $49.3 \%$ & $48.8 \%$ & $49.6 \%$ \\
\hline Day1+3 & $50.7 \%$ & $53.1 \%$ & $52.7 \%$ \\
\hline Day2+3 & $53,7 \%$ & $51,2 \%$ & $52.0 \%$ \\
\hline Day1+2+3 & $49.2 \%$ & $49.5 \%$ & $49.7 \%$ \\
\hline
\end{tabular}

By analyzing results of offline detection for different combinations of samples, it is noticeable that accuracy of detection varies from day to day. However, the combination of samples from different days results in just $1-3 \%$ percent performance drop compared to the to the worst-case performance of single sessions. Based on this, we can assume that for single session performance will be higher than the long-term performance by $5-10 \%$. Using that assumption, we will acquire only one session of data for next stages of the experiment, and it is better to conduct real-time experience within the same or next day after data acquisition session.

3) Case 3: automated labeling, reduced sampling time, signal filtering.: Next step to real-time motion classification is the automated detection of motion. The EMG signal derivative approach, described in the methods section, was tested for subject 1 and it showed its efficiency by $100 \%$ accuracy and high precision. This detection method showed the ability to discard most of the noise and it showed its advantage in comparison with methods based on WL, RMS value of the signal, and dedicated neural network. Furthermore, we visually analyzed motion contributing frequency components. All the motions were characterized with peaks at frequencies from $50 \mathrm{~Hz}$ to $100 \mathrm{~Hz}$. Based on that we implemented software bandpass filter with passing band of $30-150 \mathrm{~Hz}$. Such measure allowed us to increase the number of samples per second to 20 without the loss of accuracy. The increase of sampling frequency allowed to decrease the amount of unrelated data within the analyzed region from less than $100 \mathrm{~ms}$ to less than $50 \mathrm{~ms}$. Resultant work principle is shown in "Fig. 3".

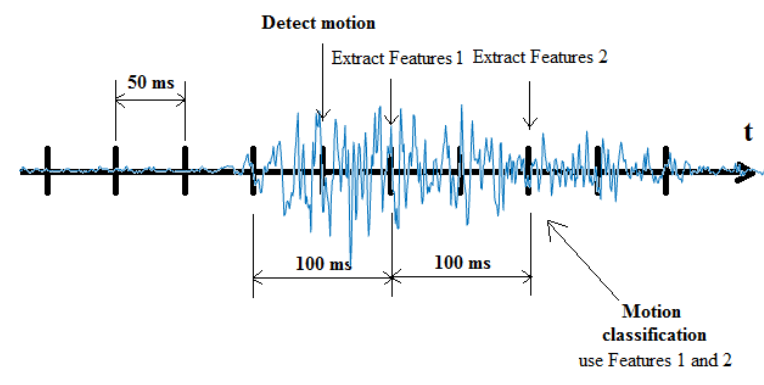

Fig. 3. Principle scheme of motion detection and classification.

The sum of the measures stated above allowed to increase the accuracy of offline classification up to $82 \%$ for 8 classes. To compare the results with previous works, we calculated accuracy of classification for 6 classes of motions (without radial and ulnar deviation), and it is resulted in $91.3 \%$. The confusion matrixes for the whole dataset for best accuracy classification of 8 and 6 classes of motions is shown in "Fig. 4".

\begin{tabular}{|c|c|c|c|c|c|c|c|c|c|}
\hline Class & 1 & 2 & 3 & 4 & 5 & 6 & 7 & 8 & \\
\hline 1 & 62 & 7 & 1 & 2 & 0 & 0 & 0 & 1 & \\
\hline 2 & 6 & 57 & 1 & 3 & 0 & 5 & 1 & 0 & \\
\hline 3 & 0 & 0 & 61 & 1 & 2 & 0 & 0 & 0 & \\
\hline 4 & 0 & 1 & 5 & 50 & 4 & 14 & 0 & 1 & \\
\hline 5 & 0 & 0 & 2 & 1 & 54 & 5 & 0 & 6 & \\
\hline 6 & 2 & 3 & 0 & 12 & 2 & 45 & 0 & 0 & \\
\hline 7 & 0 & 2 & 0 & 0 & 0 & 1 & 69 & 0 & \\
\hline 8 & 0 & 0 & 0 & 1 & 8 & 0 & 0 & 62 & \\
\hline Accuracy & \multicolumn{9}{|c|}{ 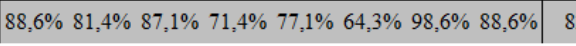 } \\
\hline & Class & 1 & 2 & 3 & 4 & 5 & 6 & \multicolumn{2}{|c|}{ Accuracy } \\
\hline & 1 & 64 & 5 & 2 & 2 & 0 & 1 & \multicolumn{2}{|c|}{$86,5 \%$} \\
\hline & 2 & 5 & 61 & 0 & 2 & 2 & 0 & \multicolumn{2}{|c|}{$87,1 \%$} \\
\hline & 3 & 0 & 0 & 66 & 4 & 0 & 0 & \multicolumn{2}{|c|}{$94,3 \%$} \\
\hline & 4 & 1 & 4 & 2 & 62 & 1 & 4 & \multicolumn{2}{|c|}{$83,8 \%$} \\
\hline & 5 & 0 & 0 & 0 & 0 & 67 & 0 & \multicolumn{2}{|c|}{$100,0 \%$} \\
\hline & 6 & 0 & 0 & 0 & 0 & 0 & 65 & \multicolumn{2}{|c|}{$100,0 \%$} \\
\hline & curacy & $91,4 \%$ & $87,1 \%$ & $94,3 \%$ & $88,6 \%$ & $95,7 \%$ & $92,9 \%$ & \multicolumn{2}{|c|}{$91,7 \%$} \\
\hline
\end{tabular}

Fig. 4. Confusion matrixes for best performance of ANN for day 3, subject 1 for 8 classes (top) and 6 classes (bottom).

4) Case 4: real-time experiment: The Last stage of the experiments is Real-time motion classification for 8 classes of motions. For that purpose, we implemented visual feedback in Blender Game Engine. The resultant system has 4 degrees of freedom, with 2 states for Open/Close, and 3 states for each pair of wrist motions. TCP/IP protocol was used to send data from MATLAB to blender. During the experiment, it was observed that system has the low accuracy of real-time signal detection in case of 6 and 8 classes of motions, however, it worked well for the case of 2-4 classes of motions (hand flexion/extension, pronation/supination). 
Pre-Print Version. To cite correctly this paper please refer to: https://ieeexplore.ieee.org/document/8311527

\section{DISCUSSION}

Based on the experimental result we conclude that for the 2-electrode system introduced in the research it is possible to detect 8 hand motions. The list of motions is following: hand close/open, wrist flexion/extension, pronation/supination, and ulnar/radial deviation. Also, it was determined that such time domain features as MAV, DMAV, ZC, WL has sufficient divergence for those types of motions to contribute to motion classification. For frequency domain, it is effective to analyze the mean frequency and its amplitude, and the frequency of the highest amplitude component. Moreover, the bandpass filter with passing band of $30-150 \mathrm{~Hz}$ has a significant beneficial effect on motion classification accuracy. In our research, we analyzed first $200 \mathrm{~ms}$ of the motion, by extracting features of two sets of data that correspond to first and second $100 \mathrm{~ms}$ of the signal. During the experiments, we achieved high accuracy of detection of the beginning of the motion by analyzing the peaks of EMG signal derivative. The real-time experiment showed high accuracy of classification for 2 and 4 classes situation for flexion/extension, and flexion/extension plus pronation/supination respectively. For higher number of classes real-time system did not show sufficient results, meanwhile, it can be explained in a long time interval from initial data acquisition and real-time experiment (more than two weeks). The worst-case delay between the beginning of motion and its recognition is $250 \mathrm{~ms}$ plus computational time (less than $15 \mathrm{~ms})$. Also, the system has relatively low computational complexity, it requires approximately 300 multiplications per sample (50ms of data) for motion detection, and approximately 1600 multiplications for feature extraction per sample $(100 \mathrm{~ms}$ of data). However, it is still should be verified is the method applicable for low-cost single-board computers in order to create computational unit embedded into the prosthesis. One more limitation of this work is that method showed the accuracy of motion recognition comparable to the state-ofart but it was tested only on one subject, further checks for repeatability should be done.

\section{CONCLUSION}

This study reports several research results. First, we developed a method for identification of the beginning of motion which showed high accuracy and reliability and proved its advantage over the other considered methods in terms of accuracy and computational simplicity. Second, we developed a method of motion classification that showed $82 \%$ accuracy for 8 classes of motions and $91.7 \%$ accuracy for 6 classes of motions when tested Offline. Finally, a real-time motion recognition system showed partial sustainability. For further improvement, there is a suggestion to implement Neural Network-adaptive wavelet analysis algorithm that was utilized in [8]. Also, it is suggested to implement hardware band-pass filter and to implement software optimization.

\section{REFERENCES}

[1] I. Vujaklija, D. Farina and O. Aszmann, "New developments in prosthetic arm systems", Orthopedic Research and Reviews, vol. 8, pp. 3139, 2016.
[2] C. De Luca, "The Use of Surface Electromyography in Biomechanics", JOURNAL OF APPLIED BIOMECHANICS, vol. 13, pp. 135-163, 1997.

[3] R. Chowdhury, M. Reaz, M. Ali, A. Bakar, K. Chellappan and T. Chang, "Surface Electromyography Signal Processing and Classification Techniques", Sensors, vol. 13, no. 9, pp. 12431-12466, 2013.

[4] G. ElKoura and K. Singh, Handrix: animating the human hand, in ACM SIGGRAPH/EG Symposium on Computer Animation 2003, SCA 03, 26-27 July 2003, San Diego, California [Online]. Available: http://www.dgp.toronto.edu/ gelkoura/handrix/paper.html. [Accessed: 15-Feb-2017].

[5] D. Graupe and W. Cline, "Functional Separation of EMG Signals via ARMA Identification Methods for Prosthesis Control Purposes",IEEE Transactions on Systems, Man, and Cybernetics, vol. -5, no. 2, pp. 252259, 1975.

[6] M. Ison, I. Vujaklija, B. Whitsell, D. Farina and P. Artemiadis, "HighDensity Electromyography and Motor Skill Learning for Robust LongTerm Control of a 7-DoF Robot Arm", IEEE Transactions on Neural Systems and Rehabilitation Engineering, vol. 24, no. 4, pp. 424-433, 2016.

[7] Ning Jiang, K. Englehart and P. Parker, "Extracting Simultaneous and Proportional Neural Control Information for Multiple-DOF Prostheses From the Surface Electromyographic Signal”, IEEE Transactions on Biomedical Engineering, vol. 56, no. 4, pp. 1070-1080, 2009.

[8] M. Arvetti, G. Gini and M. Folgheraiter, "Classification of EMG signals through wavelet analysis and neural networks for controlling an active hand prosthesis", 2007 IEEE 10th International Conference on Rehabilitation Robotics, 2007.

[9] C. Castellini and P. van der Smagt, "Surface EMG in advanced hand prosthetics", Biological Cybernetics, vol. 100, no. 1, pp. 35-47, 2008.

[10] M. Gandolla, S. Ferrante, G. Ferrigno, D. Baldassini, F. Molteni, E. Guanziroli, M. Cotti Cottini, C. Seneci and A. Pedrocchi, "Artificial neural network EMG classifier for functional hand grasp movements prediction", Journal of International Medical Research, 2016.

[11] S. Raurale, "Acquisition and processing real-time EMG signals for prosthesis active hand movements", 2014 International Conference on Green Computing Communication and Electrical Engineering (ICGCCEE), 2014

[12] S. Das Gupta, S. Kundu, R. Pandey, R. Ghosh, R. Bag and A. Mallik, "Hand Gesture recognition and classification by Discriminant and Principal Component Analysis using Machine Learning techniques", 2012. [Online]. Available: https://pdfs.semanticscholar.org/de62/ 7e1e12a2d6230d6f54f7a76c17f3bda22b11.pdf. [Accessed: 01- Mar2017].

[13] S. Bitzer and P. van der Smagt, "Learning EMG control of a robotic hand: towards active prostheses", Proceedings 2006 IEEE International Conference on Robotics and Automation, 2006. ICRA 2006.

[14] B. Crawford, K. Miller, P. Shenoy and R. Rao, "Real-Time Classification of Electromyographic Signals for Robotic Control", Proceeding of AAAI, pp. 523-528, 2005.

[15] S. Vigneswari, D. Savithri and V. Mahendran, "Gripping force measurement and EMG classification for hand functions", International Journal of Applied Engineering Research, vol. 10, no. 7, pp. 17315-17326, 2015. 